\title{
Strain rate dependence of SM490B at room temperature and application to Cowper- Symonds constitutive models
}

\author{
Taisei Sakai ${ }^{*}$, Hiroyuki Yamada ${ }^{2}$, Nagahisa Ogasawara ${ }^{2}$, Muneyuki Kokudai ${ }^{3}$, and \\ Tsutomu Fukui ${ }^{3}$ \\ ${ }^{1}$ Graduate School of Science and Engineering, National Defense Academy, Yokosuka, Japan. \\ ${ }^{2}$ National Defense Academy, Department of Mechanical Engineering, Yokosuka, Japan \\ ${ }^{3}$ Nippon Kaiji Kyokai, Research Institute, Chiyoda, Tokyo, Japan.
}

\begin{abstract}
Steel is widely used as a constituent material for various structures such as automobiles and ships. To perform high precision analysis including high strain rate behavior, an understanding for the strain rate dependence of material strength becomes very important. The purpose of this study is to evaluate the strain rate dependence of material strength with rolled steels for welded structure, JIS SM490B (ASTM E). We investigated the deformation characteristics at room temperature by performing compression tests at a wide range of strain rates and applied the obtained experimental results to the material constitutive model using an optimization method of Nelder-Mead method. The quasi-static tests were conducted using a universal testing machine at the strain rate of $10^{-3}, 10^{-2}$ and $10^{-1} \mathrm{~s}^{-1}$. The impact test was conducted using a split Hopkinson pressure bar apparatus at the strain rate of approximately $10^{3} \mathrm{~s}^{-1}$. As the results of the compression tests, it was confirmed that SM490B has a positive strain rate dependence of material strength. The Cowper-Symonds constitutive model showed good agreement with the experimental results up to the strain of $20 \%$. However, the error became larger between experimental results and CS approximation as the strain increases to $20 \%$ or more.
\end{abstract}

\section{Introduction}

It is generally known that the mechanical properties of metallic materials change with strain rate and temperature. Among various metal, steel is widely used as a constituent material for various structures such as automobiles and ships. Taking ships as an example, groundings, collisions between ships and marine structures are the situations in which strain rate and temperature effect of steels should be considered. In addition, collapse behavior caused by whipping which is a transient elastic vibration of the ship hull girder caused by wave impact loads on the hull such as slamming may be the situation as well. The elucidation of these problem requires real-scale experiments, but it is very difficult. Therefore, it is important to reproduce these phenomena by numerical analysis. The material constitutive model is

* Corresponding author: em59031@nda.ac.jp 
required for numerical analysis of impact deformation. This material constitutive model must take into account the strain rate dependence of material strength, including high strain rates. However, there are no clear rules for determining the material constants of the material constitutive model obtained from the experimental results.

The purpose of this study is to evaluate the strain rate dependence of material strength with a rolled steel for welded structure, JIS (Japanese Industrial Standards) SM490B (ASTM E). We investigated the deformation characteristics at room temperature by performing compression tests at a wide range of strain rates and applied the obtained experimental results to the material constitutive model using Nelder-Mead method (downhill simplex method) which is one of the famous optimization methods.

\section{Material and method}

\subsection{Material}

Rolled steel for welded structure, JIS (Japanese Industrial Standards) SM490B (ASTM E) was used as the test material. As the shape of the test specimen, the CC5 type (diameter 5 $\mathrm{mm}$, height $4 \mathrm{~mm}$ ) specified in JIS Z 2205:2019 was used for all tests.

\subsection{Method}

\subsubsection{Quasi-static test}

The quasi-static test was conducted using a universal testing machine at room temperature. The strain rates were chosen in the range of $10^{-3}$ to $10^{-1} \mathrm{~s}^{-1}$.

\subsubsection{Impact test}

The impact test was conducted using a split Hopkinson pressure bar (SHB) apparatus at room temperature. The strain rate was approximately $10^{3} \mathrm{~s}^{-1}$. Figure 1 shows the schematic diagram of SHB apparatus. The SHB method $[1,2]$ is as a way to measure stress and strain by the stress wave propagating through two elastic bars. The test specimen is sandwiched between these elastic bars.

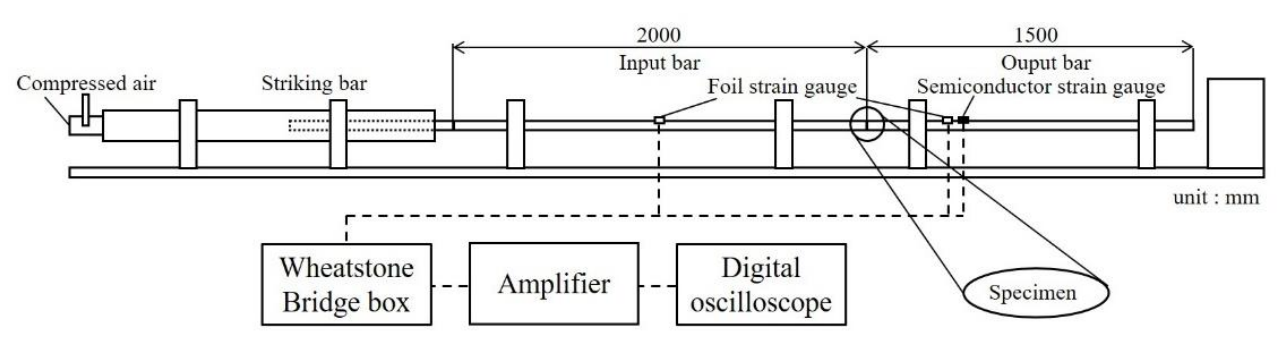

Fig. 1. Schematic diagram of split Hopkinson pressure bar. 
An elastic stress wave is generated by impacting the striking bar with the end of the input bar. When this elastic wave reaches the specimen, this is deformed and its information is transmitted to the output bar. The remaining part is reflected on the input bar. When the specimen is in dynamic stress equilibrium, the nominal stress, strain and strain rate are expressed as the following equation by one-dimensional wave equation based on elastic wave propagation theory.

$$
\begin{aligned}
& \sigma(t)=\frac{1}{A_{s}} A E \varepsilon_{T}(t) \\
& \varepsilon(t)=\int_{0}^{t} \dot{\varepsilon}(t) \mathrm{dt} \\
& \dot{\varepsilon}(t)=\frac{2 C}{L_{s}}\left[\varepsilon_{\mathrm{I}}(t)-\varepsilon_{T}(t)\right]
\end{aligned}
$$

where $A$ is cross-section area of the bar, $A_{S}$ is cross-section area of the specimen, $E$ is Young's modulus of bars, $C$ is elastic wave velocity, $L_{S}$ is length of specimen, $\varepsilon_{\mathrm{I}}$ is strain of incident wave and $\varepsilon_{T}$ is strain of transmitted wave.

\section{Constitutive models and its determination method}

\subsection{Cowper-Symonds model}

Cowper-Symonds (CS) model [3] is one of the most famous constitutive models in consideration of strain rate dependence of material strength. CS model is based on theory that viscous part of flow stress is proportional to strain rate and expressed as the following equation.

$$
\sigma=\sigma_{s}\left[1+\left(\frac{\dot{\varepsilon}}{D}\right)^{m}\right]
$$

where $D$ and $m$ are material constants and $\sigma_{S}$ is quasi-static flow stress. In this study, $\sigma_{S}$ is given by a Holloman equation [4] and defined as follows.

$$
\sigma_{S}=B \varepsilon^{n}
$$

where $B$ and $n$ are material constants.

\subsection{Optimization method}

In this study, the optimization by Nelder-Mead method (downhill simplex method) was used in determining the material constants of constitutive model. Nelder-Mead method is one of the non-linear optimization methods. It is also called downhill simplex method, amoeba method, or polytope method. This method was first suggested by Himsworth, Spendley and Hext [5]. And then, Nelder and Mead [6] refined this. The main advantages of Nelder-Mead method are that the derivative is unnecessary and the algorithm is very simple. This method uses the algorithm of a simplex and repeats 4 steps called Reflection, Expansion, Contraction and Shrink like an amoeba to search optimum solutions for set objective function.

In this study, the objective function was sum of squared residuals between the stress of the experimental results and CS model at each strains. Minimizing this objective function using above mentioned optimization method, material constants of constitutive model were 


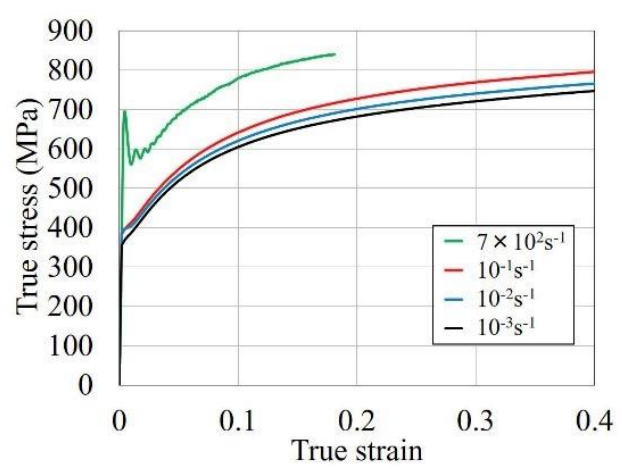

Fig. 2. True stress-true strain relations for SM490B.

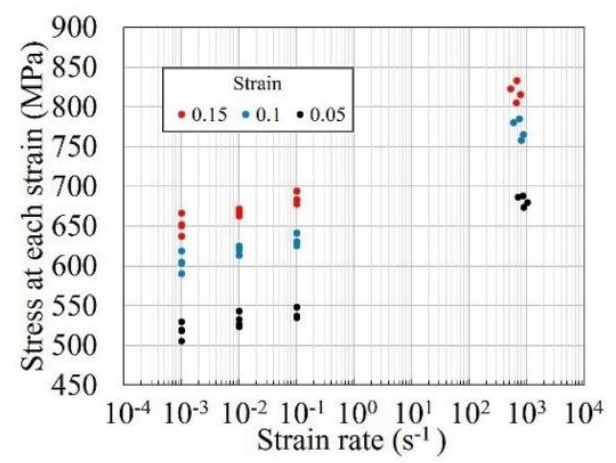

Fig. 3. Stress at each strain-stress rate relations for SM490B.

determined. However, since the experimental results included the yield phenomenon, the optimizations were calculated after the yield point, namely, in plastic region. The calculations are conducted by MATLAB.

\section{Results and discussion}

\subsection{Experimental results}

Figure 2 shows the typical stress-strain relations at each strain rates. In addition, the relationship between stress at each strain and strain rate is shown in Figure 3. These show that the flow stress increases as the strain rate increases. Therefore, the material strength of SM490B has a positive strain rate dependence.

\subsection{Application to constitutive models}

To express the strain rate dependence of material strength for SM490B as the constitutive model, the material constants of CS model were decided using the optimization method described in chapter 3 . Table 1 shows the summary of the determined material constants. The condition (1) was determined by both quasi-static and impact test between strain 0.01 and 0.18 , condition (2) was determined by only quasi-static test between strain 0.01 and 0.18 , condition (3) was determined by only quasi-static test between strain 0.01 and 0.4 . Note that condition (1) excludes the strain rate of $10^{-2} \mathrm{~s}^{-1}$ since a convergent solution could not be obtained by the Nelder-Mead method when referring to the experimental results of this strain rate. Figure 4 shows true stress-true strain relations in comparison with the experimental results and the CS model of condition (1). The CS model were in good agreement with the experimental results at low strain after the yield point (approximately 20\%). However, the error become larger between experimental results and CS model as the strain increases. This is because the Holloman equation cannot express the experimental results as the strain increases. It will be necessary to modify the expression of $\sigma_{S}$ in the future. In addition, it is considered that the adiabatic temperature rise occurs due to effect of high strain rate deformation. Therefore, it will be required to use the constitutive model which can consider the temperature dependence such as Johnson-Cook (JC) model [7]. Therefore, it is needed to experimentally clarify the effects of strain rate and temperature on strength of SM490B in the future. 
Table 1. Material constants of CS model.

\begin{tabular}{|c|c|c|c|c|c|}
\hline \multirow{2}{*}{ Condition } & \multirow{2}{*}{$\begin{array}{c}\text { Referenced data } \\
\text { Range of strain }\end{array}$} & \multicolumn{4}{|c|}{ Material constants } \\
\cline { 3 - 6 }$(1)$ & $\begin{array}{c}\text { Quasi-static and impact } \\
0.01 \text { to } 0.18\end{array}$ & 818.9 & 0.1888 & 3024000 & 0.09318 \\
\hline$(2)$ & $\begin{array}{c}\text { Quasi-static } \\
0.01 \text { to } 0.18\end{array}$ & 891.6 & 0.2083 & 351700 & 0.1234 \\
\hline$(3)$ & $\begin{array}{c}\text { Quasi-static } \\
0.01 \text { to } 0.4\end{array}$ & 539.9 & 0.1785 & 285.3 & 0.03309 \\
\hline
\end{tabular}

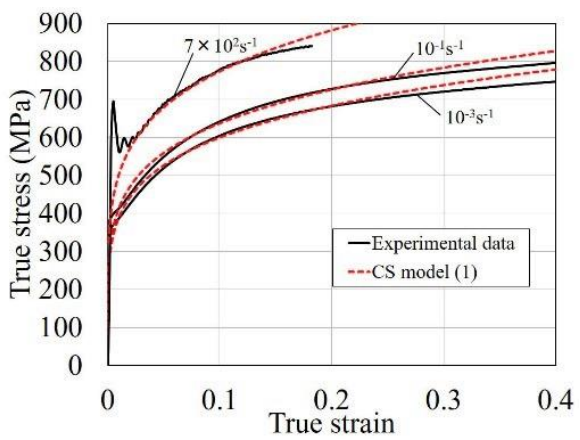

Fig. 4. Comparison between experimental results and CS model of condition (1) in stressstrain relations of SM490B.

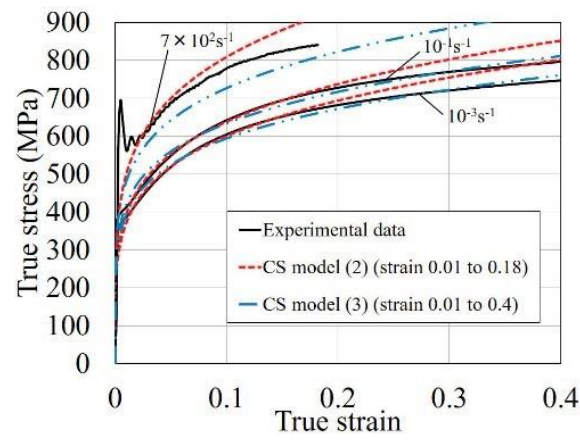

Fig. 5. Comparison between experimental results and CS model with referenced by the only quasi-static test in stress-strain relations of SM490B.

Table 2. Error rate between experimental results and CS model

\begin{tabular}{|c|c|c|c|c|c|}
\hline \multirow{2}{*}{ Condition } & \multirow{2}{*}{$\begin{array}{c}\text { Referenced data } \\
\text { Range of strain }\end{array}$} & \multicolumn{4}{|c|}{ Error rate $[\%]$} \\
\cline { 3 - 6 } & & $10^{-3}\left[\mathrm{~s}^{-1}\right]$ & $10^{-2}\left[\mathrm{~s}^{-1}\right]$ & $10^{-1}\left[\mathrm{~s}^{-1}\right]$ & $7 \times 10^{2}\left[\mathrm{~s}^{-1}\right]$ \\
\hline$(1)$ & $\begin{array}{c}\text { Quasi-static and impact } \\
0.01 \text { to } 0.18\end{array}$ & 1.7 & & 1.6 & 1.4 \\
\hline$(2)$ & $\begin{array}{c}\text { Quasi-static } \\
0.01 \text { to } 0.18\end{array}$ & 2.7 & 2.7 & 2.5 & 4.6 \\
\hline$(3)$ & $\begin{array}{c}\text { Quasi-static } \\
0.01 \text { to } 0.4\end{array}$ & 1.4 & 1.4 & 1.4 & 6.2 \\
\hline
\end{tabular}

Table 2 shows the error rate by arithmetic mean of residual error at each conditions. The calculation of the error rate is as follows.

$$
\text { Error rate [\%] }=\frac{1}{N} \sum_{i=\varepsilon_{\min }}^{\varepsilon_{\max }} \frac{\left|\sigma_{m i}-\sigma_{T i}\right|}{\sigma_{T i}} \times 100
$$

where $N$ is the total numbers of the data, $\varepsilon_{\min }$ is minimum strain, $\varepsilon_{\max }$ is maximum strain, $\sigma_{T}$ is experimental stress and $\sigma_{m}$ is stress by constitutive model. The condition (1) had the low error rate compared to other conditions. Comparing the conditions (2) and (3) in which the CS model was predicted only in the quasi-static test, the error rate in the quasi-static test is 
smaller when the reference strain amount is larger. However, the error rate of the impact test is smaller when the reference strain amount is smaller.

The effect of the error shown in Table 2 with the stress-strain curve was confirmed. Figure 5 shows comparison between experimental results and CS model which refer to the only quasi-static test (reference strain 0.01 to 0.18 and 0.01 to 0.4 ) in the stress-strain relations. In the Figure 5, the stress-strain relation for the strain rate of $10^{-2} \mathrm{~s}^{-1}$ is omitted for the sake of clarity. The CS model by condition (2) was good agreements with the quasi-static tests up to $20 \%$ strain. However, it corresponded only at an early stage of deformation in the impact tests. On the other hand, the model by condition (3) fit to the quasi-static tests, but this cannot express the impact tests. These show that it is difficult to predict the experimental result by constitutive model obtained by Nelder-Mead method from only quasi-static test. Furthermore, it is also essential to compare Nelder-Mead method with another optimization method in the future.

\section{Conclusions}

To investigate the deformation characteristics of SM490B at room temperature, the compression tests were conducted at the wide range of strain rate including impact deformation. The results indicate that the material strength of SM490B has a positive strain rate dependence. The experimental results were good agreement with the CS model by Nelder-Mead method at low strain. However, the error become larger between experimental results and CS model as the strain increases. It will be necessary to modify the expression of $\sigma_{S}$ in the future. Furthermore it also will be required to use the constitutive model which can consider the temperature dependence such as JC model. The result of comparison with the difference of referenced data shows that it is difficult to express the experimental result by constitutive model from only quasi-static test. Therefore, the importance of high strain rate experiments was reconfirmed. The CS model of SM490B obtained from the optimization at a wide range of strain rates by Nelder-Mead method provide a useful tool in the field of the impact problem for ships.

\section{References}

1. H. Kolsky, An Investigation of the Mechanical Properties of Materials at Very High Rates of Loading, in Proceedings of the Physical Society B, 62, 676-700 (1949)

2. B. Hopkinson, Philos. Trans. R. Soc. London A, 213, 437-456 (1914)

3. G.R. Cowper, P.S. Symonds, Strain-hardening and strain rate effects in the impact loading of cantilever beams, Brown University Division of Applied Mathematics Report, 28 (1957)

4. J. H. Hollomon, Trans. AIME, 162, 268-290 (1945)

5. W. Spendley, G. R. Hext, F. R. Himsworth, Technometrics, 4, 441-461 (1962)

6. J. A. Nelder, R. Mead, The Computer Journal, 7, 308-313 (1965)

7. G.R. Johnson, W. H. Cook, A Constitutive Model and Data for Metals Subjected to Large Strains, High Strain Rates, and High Temperatures, in Proceedings of the Seventh International Symposium on Ballistic, 19-21 April 1983, The Hague, The Netherlands, 541-547 (1983) 